\title{
CONSIDERAÇÕES ACERCA DA AVALIAÇÃO PSICOLÓGICA DAS COMORBIDADES PSIQUIÁTRICAS EM OBESOS
}

\author{
Fabiana Silva Costa \\ Denise Ruschel Bandeira \\ Clarissa Trentini" \\ Mirian Brilmann ${ }^{\infty}$ \\ Rogério Friedman ${ }^{\mathbb{E}}$ \\ Maria Angélica Nunes ${ }^{\Phi}$
}

\begin{abstract}
RESUMO. Pretende-se neste artigo apresentar uma revisão teórica sobre avaliação psicológica, obesidade e comorbidades psiquiátricas mais frequentes na população obesa. A obesidade é uma doença clínica de múltiplas causas, e atualmente é considerada uma epidemia; assim seu adequado entendimento deve envolver uma equipe composta por diversos profissionais de saúde, incluindo o psicólogo. Serão abordadas questões pertinentes ao tema de obesidade, como etiologia, incidência e a relação entre obesidade e transtornos psiquiátricos. Serão propostos alguns instrumentos com o objetivo de estruturar informações necessárias ao tratamento e à demanda do paciente obeso, e reflexões referentes à avaliação psicológica em obesos e a prática do profissional de psicologia.
\end{abstract}

Palavras-chave: Obesidade; avaliação psicológica; transtorno psiquiátrico.

\section{CONSIDERATION ABOUT PSYCHOLOGICAL ASSESSMENT OF PSYCHIATRIC CO MORBIDITY IN OBESE}

\begin{abstract}
We intend in this article to present a theoretical review about psychological assessment, obesity and psychiatric co morbidity. Obesity is a clinical illness, it has multi factorial causes, and nowadays it is considered an epidemic, so the right knowledge must involve all health professional, include the psychologist. In this article we talk about something related to obesity, like etiology, prevalence, obesity and mental disorder. We suggest some instruments to structure essential information related to the treatment and the obese patient objective, and we reflect about psychological assessment in obesity and the psychologist professional practice.
\end{abstract}

Key words: Obesity; psychological assessment; mental disorder.

\section{CONSIDERACIONES SOBRE LA EVALUACIÓN PSICOLÓGICA DE LAS COMORBIDADES PSIQUIÁTRICAS EN OBESOS}

RESUMEN. Se pretiende en ese artículo presentar una revisión teórica sobre la evaluación psicológica, obesidad y comorbidades psiquiátricas más frecuentes en esa población. La obesidad es una enfermedad clínica con diversas causas y actualmente es considerada una epidemia, así el adecuado entendimiento de la obesidad debe involucrar un equipo compuesto por diversos profesionales de salud, incluso el psicólogo. En ese artículo serán abordadas cuestiones pertinentes a la temática de la obesidad, como etiología, prevalencia y la relación entre obesidad y los trastornos psiquiátricos. Serán propuestos algunos instrumentos* con el objetivo de estructurar informaciones necesarias al tratamiento y a la demanda del paciente obeso, reflexiones referentes a la evaluación psicológica en obesos y la práctica del profesional de Psicología.

Palabras-clave: Obesidad; evalución psicológica; trastorno psiquiátrico.

\footnotetext{
* Psicóloga e mestranda do Programa de Pós-Graduação em Endocrinologia da Universidade Federal do Rio Grande do Sul-UFRGS.

* Psicóloga, Doutora em Psicologia, Professora do Instituto de Psicologia da UFRGS.

II Psicóloga, Doutora em Psiquiatria, Professora do Instituto de Psicologia da UFRGS.

æ Psicóloga, Mestre em Psicologia.

Æ Médico Endocrinologista, Doutor em Endocrinologia, Professor da Faculdade de Medicina da UFRGS.

Ф Médica, Doutora em Epidemologia, Professor do programa de Pós-Graduação em Epidemologia, UFRGS.
} 


\section{OBESIDADE VISUALIZANDO}

A obesidade constitui-se em um problema de saúde pública com etiologia multifatorial, sendo definida como resultante da associação entre a vulnerabilidade genética e um ambiente facilitador (Greenway \& Smith, 2000; Dobrow, Kamenetz \& Devlin, 2002). A obesidade está relacionada com a morbidade e mortalidades aumentadas. As doenças frequentemente associadas são: hipertensão, dislipidemia, diabetes mellitus, doenças cardiovasculares, osteoartrite, apnéia do sono, alguns tipos de câncer (cólon, mama, endométrio, bexiga), distúrbios menstruais, infertilidade e risco gestacional (Devlin, Yanovski \& Wilson, 2000).

A dimensão dos prejuízos causados pela obesidade é causa de crescente preocupação. Nos Estados Unidos, 1/3 dos adultos (27.6\% dos homens e $33,2 \%$ das mulheres) se constituía de obesos. Dois por cento dos homens adultos e seis por cento das mulheres adultas são obesos grau III (Altfas, 2002; Antone, Lord, Demeester \& Crookes, 2003; Baskin, Ard, Frankli \& Allison, 2005).

Nos países da América Latina observa-se uma variação na incidência de obesidade de $10 \%$ a $35 \%$, sendo que aos equatorianos corresponde menor índice e aos paraguaios o maior (Braguinsky, 2002). No Brasil, estudos realizados entre 1975 e 1989 já indicavam um aumento de $70 \%$ de casos de obesidade, sinalizando que o excesso de peso se tornaria um problema mais prevalente que o déficit nutricional (Dobrow et al., 2002). Publicação recente do IBGE - Instituto Brasileiro de Geografia e Estatística (2004) - apresentou um preocupante panorama da população adulta brasileira, com índice de $40 \%$ dos indivíduos com sobrepeso. Dentre os indivíduos com excesso de peso, 8,9\% são homens obesos e 13,1\% são mulheres obesas. Na Região Sul, a incidência de obesidade é de $10,1 \%$ entre os homens e $15,1 \%$ entre as mulheres.

A realidade dos níveis elevados de obesidade em diversos países do mundo motivou instituições como a International Obesity Task Force e a Organização Mundial de Saúde a declarar que a obesidade é uma epidemia de proporções globais (Tauber, 1998). Tal decisão reforça a importância de os profissionais atentarem para o diagnóstico da obesidade.

Definir o que é obesidade não é tarefa simples. $\mathrm{O}$ método diagnóstico mais utilizado em pesquisas tem sido o IMC (índice de massa corporal). O IMC é obtido através da seguinte fórmula: peso/altura $\mathrm{x}$ altura $\left(\mathrm{Kg} / \mathrm{m}^{2}\right)$, sendo assim classificado: < 18,5 (baixo peso), 18,5 - 24,9 (peso normal), 25 - 29,9 (sobrepeso), 30-34,9 (obesidade I), 35 - 39,9 (obesidade II), $\geq 40$ (obesidade III) (Segal,
2004). Quanto maior o IMC, nas faixas de sobrepeso à obesidade III, maiores são as chances de complicações clínicas. Contudo, essa classificação não abrange toda a complexidade dessa doença: dispêndio energético, variação de massa gorda e magra, entre outras variáveis.

Na CID-10 (Organização Mundial da Saúde, 1993), por exemplo, a obesidade é classificada como uma condição clínica geral. No DSM-IV-TR (American Psychiatric Association, 2002) não está classificada como transtorno psiquiátrico, por não estar associada consistentemente a uma síndrome psicológica ou comportamental. Em seu projeto inicial, o DSM incluía, além da anorexia nervosa e da bulimia nervosa, o transtorno da compulsão alimentar periódica (TCAP), muito frequente entre os pacientes obesos. Atualmente esta categoria diagnóstica ficou restrita ao apêndice B da classificação (transtornos sem outras especificações), na tentativa de melhor definir e estudar este problema.

Questiona-se se o TCAP seria uma categoria diagnóstica distinta, visto que abrange um grupo heterogêneo de pacientes que não preenchem critérios para anorexia nervosa (AN) e bulimia nervosa (BN), ou que apresentam variações dessas patologias, por exemplo, preenchendo critérios para bulimia nervosa, exceto pelo mecanismo compensatório (Wonderlich, Joiner, Keel, Williamson \& Crosby, 2007).

Fairburn, Cooper, Doll, Norman e O'Connor (2000) defendem que não há constância no estabelecimento dos sintomas de TCAP, e mostram isso num estudo com 102 pacientes com bulimia nervosa e 48 com TCAP num seguimento de cinco anos. Após cico anos $51 \%$ dos pacientes com bulimia nervosa continuam a preencher critérios para $\mathrm{BN}$ ou outro transtorno alimentar. Dos pacientes com TCAP, apenas $18 \%$ preenchem critérios para transtorno alimentar, sendo somente $4 \%$ para TCAP.

Pope et al., (2006), no entanto, encontraram outro resultado em uma pesquisa com 300 pacientes obesos ou com sobrepeso (150 com TCAP e 150 sem TCAP) e seus familiares em $1^{\circ}$ grau (888 familiares). Os pesquisadores buscaram avaliar a estabilidade do diagnóstico de TCAP e encontraram uma duração média do curso da doença de 14,4 anos, sugerindo que o TCAP teria um curso tão crônico quanto a AN e BN. Diante de resultados ainda contraditórios, o questionamento da relação entre transtorno psiquiátrico, transtorno alimentar e obesidade ainda permanece.

\section{TRANSTORNO PSIQUIÁTRICO E OBESIDADE}

Tanto os fatores biológicos e comportamentais como os aspectos psicológicos são de fundamental importância para o entendimento e manejo clínico do 
paciente obeso (Dobrow et al., 2002). Alguns estudos, como o de Kendrick (1996), Carpenter, Hansin, Alisson e Faith (2000), Becker, Margraf, Turke, Soeder e Neumer (2001), Strawbridge, Deleger, Roberts e Kaplan (2002) e Sturm (2001), documentam a maior incidência de psicopatologia em obesos, mas há muitos desentendimentos quanto à sua extensão e natureza. Essas discrepâncias ocorrem por serem estudos de amostras regionais, com idade restrita a pequenas faixas etárias, com apenas mulheres, ou diagnósticos não baseados no DSM-IV-TR. (Maddi, Knoshaba, Persico, Bleecker \& VanArsdall, 1997; Wilfley, Schawartz, Spurrell \& Fairburn, 2000; John, Meyer, Rumpf \& Hapke, 2005).

De acordo com Fabricatore e Wadden (2004), a maioria das pesquisas de base populacional em obesidade não apresenta associação direta entre obesidade e psicopatologia. Não há consistente associação com transtorno depressivo maior (TDM) ou qualquer característica de personalidade. No entanto mulheres obesas, obesos classe III e comedores compulsivos têm maior risco de desenvolver transtornos psiquiátricos (Roberts, Deleger, Strawbridge \& Kaplan, 2003).

O episódio de ingestão alimentar compulsiva refere-se ao intenso e incontrolável ato de comer grandes quantidades de alimento em curto espaço de tempo, constituindo-se assim numa importante variável para o entendimento e tratamento deste problema. A presença ou ausência de TCAP define se o diagnóstico de obesidade é clínico ou com comorbidade psicopatológica (Claudino \& Borges, 2002).

Estudos epidemiológicos constatam que cerca de $30 \%$ dos pacientes obesos que procuram tratamentos para emagrecer são portadores do transtorno da compulsão alimentar periódica (TCAP), sendo que os demais obesos apresentam outros padrões alimentares, como, por exemplo, hiperfagia, e representam uma população clinicamente diferente. É importante ressaltar que indivíduos com diagnóstico de TCAP apresentam diversos graus de obesidade, já que o diagnóstico clínico não é limitado a indivíduos com sobrepeso. Obesos com TCAP constituem uma subpopulação que possui uma resposta menos efetiva aos tratamentos comportamentais convencionais para perda de peso e apresenta altos índices de transtornos do humor e de ansiedade (Yanovsky, Nelson, Dubbert \& Spritzer, 1993).

Matos e Zanella (2002) mostraram que, em uma amostra de 50 pacientes obesos classe III, 36\% tinham o diagnóstico de TCAP e 54\% apresentavam episódios de compulsão alimentar. Concluíram que há uma associação direta entre ansiedade como traço de personalidade e TCAP. Dentre os pacientes estudados, 46\% apresentaram ocorrência dessas duas condições. Destes, 76\% apresentaram preocupação com a sua imagem corporal, sendo que quanto maior a frequência de episódios de compulsão alimentar, maior a preocupação com a imagem corporal.

Pacientes com TCAP, em comparação com indivíduos obesos sem TCAP, desenvolvem a obesidade mais precocemente e apresentam maior número de tentativas de redução de peso por meio de dietas. Além da baixa autoestima, preocupam-se mais com o peso e a forma física. Os índices de transtornos psiquiátricos são mais elevados nesta população (Dobrow et al., 2002).

Duchesne (1995) menciona a importância (em função do prognóstico) de fatores como a depressão, a ansiedade e a compulsão nos quadros de Obesidade e Transtorno da Compulsão Alimentar Periódica (TCAP), sendo essas as comorbidades mais amplamente investigadas. A compulsão alimentar é frequentemente desencadeada por estados de humor disfóricos, estados ansiosos, intensa fome e grande dificuldade no controle dos impulsos. Pacientes portadores desse problema apresentam baixa autoestima e aumento da ansiedade interpessoal, percebendo-se como inadequados tanto no seu funcionamento social como no pessoal. Possuem significativo comprometimento da saúde, sendo que com frequência se observa a comorbidade de transtornos de personalidade.

Matos e Zanella (2002), pesquisando 50 pacientes obesos grau III, encontraram importante presença de sintomas depressivos, acometendo $100 \%$ dos pacientes, sendo $84 \%$ com sintomatologia grave. A ansiedade caracterizada como traço de personalidade correspondeu a 70\%, e a ansiedade situacional, a 54\%.

Entre as comorbidades psiquiátricas no transtorno alimentar está o TDAH. Um estudo realizado no Instituto de Psiquiatria da UFRJ, com 107 pacientes que buscaram o centro especializado em Transtorno de Déficit de Atenção com Hiperatividade (TDAH), mostra que $10,4 \%$ dos 86 pacientes com diagnóstico positivo para TDAH apresentaram comorbidades com transtornos alimentares (TA), com índices acima do esperado para a população geral (Mattos et al., 2004). Tal associação, entretanto, merece ser mais bem investigada.

Outro campo de investigação diagnóstica é a Síndrome do Comer Noturno, evidenciando uma incidência de $1,5 \%$ na população geral e de $10 \%$ na população ambulatorial de obesos. Essa taxa aumenta para $27 \%$ ao serem investigados pacientes candidatos 
à cirurgia da obesidade. A Síndrome do Comer Noturno é caracterizada por anorexia matinal, excesso de ingestão alimentar no período vespertino e noite e insônia acompanhada de ingestão alimentar, chegando a totalizar 56\% da sua ingestão calórica total (Dobrow et al., 2002).

Conforme referido acima, as comorbidades psiquiátricas não são raras em obesos que buscam tratamento, e isto se dá, especialmente, em função da vulnerabilidade de sua condição. Além disso, a possibilidade de um paciente obeso vir a morrer precocemente é de $50 \%$ a $100 \%$ maior que um indivíduo de peso normal. Embora a perda de peso seja considerada vital para a saúde de um obeso, ela pode provocar uma experiência estressante acompanhada de flutuações de humor. Parte desse estresse advém da necessidade (às vezes urgência) de mudar os comportamentos alimentares e as atitudes referentes à ingestão alimentar, que demandam comportamentos compensatórios de autocontrole para restabelecer uma harmonia, o qual, por sua vez, mobiliza emoções difíceis de controlar e entender. Neste sentido, questionários, inventários e escalas de autorrelato possibilitam uma reflexão e conhecimento das forças internas que atuam no comportamento alimentar (Womble \& Williamsom, 2002; Pareja, 2002).

\section{AVALIAÇÃO PSICOLÓGICA NA OBESIDADE}

Os transtornos psicológicos manifestam-se por meio de um conjunto complexo de sintomas e queixas expressas de forma subjetiva, sendo muitas vezes de difícil compreensão para o profissional que pretenda realizar uma intervenção. Assim, a utilização e o desenvolvimento de técnicas e escalas de mensuração podem conferir maior confiabilidade e validade ao diagnóstico, permitindo que sejam respondidas as hipóteses de forma mais objetiva e científica e menos intuitiva (Crippa, Hallar, Vilela, Loureiro \& Zuardi, 1999).

As considerações finais acerca de um processo de avaliação psicológica possibilitam um maior entendimento de cada indivíduo identificado, em particular. Desta forma, a inclusão de tal prática em protocolos sistemáticos de atendimento pode trazer um arsenal de informações que melhor elucidam o caso. A contribuição se dá não somente na descrição, mas também no entendimento e diagnóstico emocional do caso. Entender ou compreender os processos envolvidos em cada caso facilita a geração de hipóteses quanto ao prognóstico e intervenção, e mesmo o monitoramento do paciente (Pasquali, 2001).
O processo de avaliação psicológica também pode ser bastante útil no estabelecimento de diagnósticos diferenciais e comorbidades existentes e, embora se considere a entrevista clínica o nosso melhor instrumento, a construção de um diagnóstico apresenta-se, na maior parte das vezes, complexa. O uso de instrumentos com reconhecidas propriedades psicométricas é um importante aliado nessa tarefa (Cunha, 2000).

Dentro deste contexto, é relevante destacar que a obesidade é uma doença física, e que obesos em geral não possuem mais psicopatologias comparativamente aos não-obesos. Uma constatação feita através de pesquisas nesta área, contudo, mostra que obesos que buscam atendimento, os quais constituem a amostra da maior parte das pesquisas realizadas, apresentam marcado sofrimento e um aumento na incidência de transtornos psiquiátricos, em especial os transtornos de humor e transtornos alimentares, e merecem criteriosa avaliação física e psicológica.

De acordo com Dobrow et al. (2002), embora o manejo das condições psicopatológicas seja fundamental para o tratamento desses pacientes, é inadequado entender a obesidade como uma dificuldade de lidar com o próprio desejo ou mesmo como um problema psicológico de antemão. A tarefa diagnóstica e a de prescrição de tratamento devem ser alicerçadas em informações obtidas em entrevistas clínicas e numa bateria de questionários de avaliação, entre outros recursos disponíveis, que possibilitam informações detalhadas sobre a presença e gravidade dos sintomas. A utilização de instrumentos diagnósticos possibilita ao paciente e ao profissional identificar comportamentos que, muitas vezes por inibição do próprio paciente, são omitidos na entrevista clínica (Cordás \& Neves, 1999; Freitas, Gorenstein \& Appolinário, 2002; Parahoo, 2005).

Para a avaliação psicológica de obesos têm-se à disposição instrumentos específicos (próprios para aquela população) e genéricos (próprios, entre outras populações, para a população de obesos). De modo mais detalhado, os instrumentos específicos são projetados para investigar especificidades das doenças. No que se refere à obesidade, podem-se utilizar tanto instrumentos que avaliam o comportamento alimentar do paciente quanto fatores $\mathrm{e}$ estressores psicossociais. Os genéricos, por sua vez, são utilizados para uma variedade de doenças crônicas e podem abranger um espectro amplo de características e sintomas presentes em diversas doenças. Os instrumentos genéricos também podem ser utilizados para correlações com outras patologias e para comparar aspectos encontrados na obesidade e na 
osteoartrite no joelho, para citar um exemplo (Le Pen, Levy, Loos, Banzet \& Basdevant, 1998).

No Brasil, poucos são os instrumentos específicos disponíveis para a avaliação de condições psicológicas que podem estar associadas à obesidade, mas literatura internacional dispõe de uma série de instrumentos específicos. Tal achado aponta para a necessidade de adaptação, normatização e validação de instrumentos para o nosso meio. Para ilustrar o que foi dito, neste trabalho foi feito um levantamento dos instrumentos utilizados em pesquisas publicadas no
International Journal of Obesity, no período de 2000 a 2005. Na tabela a seguir estão alguns instrumentos, subdivididos em genéricos e específicos, sendo alguns validados e outros com necessidade de adaptação e validação.

Conforme pode ser observado nas tabelas 1 e 2 , existem instrumentos diagnósticos e de avaliação da qualidade de vida para serem usados em diversos casos (tabela 1) específicos, que avaliam especialmente a ocorrência e intensidade de comportamentos próprios da obesidade (Tabela2).

\section{Instrumentos genéricos}

Tabela 1. Instrumentos Genéricos

\begin{tabular}{|c|c|c|c|c|}
\hline & Autor & Ano & Objetivos & $\begin{array}{l}\text { Referência de } \\
\text { Validação }\end{array}$ \\
\hline $\begin{array}{l}\text { a.1) Medical Outcomes Study } 36 \text { - Item } \\
\text { Short Form (SF 36) }\end{array}$ & $\begin{array}{l}\text { Ware, Snow, Kosinski, \& } \\
\text { Gandek }\end{array}$ & 1993 & $\begin{array}{l}\text { Avalia qualidade de vida } \\
\text { referente à saúde geral }\end{array}$ & Ciconelli, 1997 \\
\hline $\begin{array}{l}\text { a.2) Mini International Neuropsychiatry } \\
\text { Interview (M.I.N.I.) }\end{array}$ & Sheehan et al.. & 1997 & $\begin{array}{l}\text { Investigação diagnóstica } \\
\text { baseada no DSM IV }\end{array}$ & Amorin, 2000 \\
\hline $\begin{array}{l}\text { a.3) Structured Clinical Interview for DSM } \\
\text { IV (SCID) }\end{array}$ & First et al. & 2002 & $\begin{array}{l}\text { Investigação diagnóstica } \\
\text { baseada no DSM IV }\end{array}$ & Versani, 1996 \\
\hline
\end{tabular}

\section{Instrumentos específico}

Tabela 2. Instrumentos Específicos

\begin{tabular}{|c|c|c|c|c|}
\hline & Autor & Ano & Objetivos & Referência de Validação \\
\hline $\begin{array}{l}\text { b.1) Escala de Compulsão Alimentar } \\
\text { Periódica (ECAP) }\end{array}$ & Gormally & 1982 & $\begin{array}{c}\text { Avalia a presença de compulsão } \\
\text { alimentar. }\end{array}$ & Freitas e Apolinário, 2001 \\
\hline $\begin{array}{l}\text { b.2) Bulimic Inventory Test Edinburg } \\
\text { (BITE) }\end{array}$ & Smith e Thelen & 1984 & $\begin{array}{l}\text { Diferencia indivíduos com bulimia, } \\
\text { anorexia e transtorno alimentar } \\
\text { ausente. }\end{array}$ & $\begin{array}{c}\text { Foi somente adaptado para o } \\
\text { português por Cordas e } \\
\text { Neves, } 1993\end{array}$ \\
\hline b.4) Três Fatores Alimentares (TFA) & Stunkard e Messick & 1985 & $\begin{array}{c}\text { Suas três subescalas avaliam } \\
\text { Restrição Cognitiva, Desinibição } \\
\text { (perda de controle) e Fome percebida. }\end{array}$ & Não está validado no Brasil \\
\hline $\begin{array}{l}\text { b.3)Body Image Assessment for Obese } \\
\text { (BIA-O) }\end{array}$ & Wiliamson & 2000 & $\begin{array}{l}\text { Avalia percepção de imagem } \\
\text { corporal, incluindo a silhueta de } \\
\text { muito magra a superobesa. }\end{array}$ & Não está validado no Brasil. \\
\hline b.5) Fat Phobia Short Form & $\begin{array}{l}\text { Bacons, Scheltema e } \\
\text { Robinson }\end{array}$ & 2001 & $\begin{array}{l}\text { São situações subdivididas em } \\
\text { subescalas sobre o sentimento de ser } \\
\text { obeso e os preconceitos envolvidos }\end{array}$ & Não está validado no Brasil. \\
\hline $\begin{array}{l}\text { b.6) The Obesity Related Problems } \\
\text { Scale (OP) }\end{array}$ & Karlsson et al. & 2003 & $\begin{array}{l}\text { Medir o impacto da obesidade na } \\
\text { atividade social. }\end{array}$ & Não está validado no Brasil. \\
\hline
\end{tabular}

\section{CONSIDERAÇÕES FINAIS}

A realização de uma avaliação psicológica criteriosa é fundamental para posterior atendimento clínico. Para tanto deve-se ter o cuidado de escolher técnicas, testes e escalas que atendam à demanda da patologia investigada e também do paciente. Por mais que se possa sugerir a utilização de alguns instrumentos para a avaliação do paciente obeso, o olhar criterioso do profissional que realiza a avaliação é imprescindível para que se construam hipóteses pertinentes.

Nada substitui o adequado conhecimento das repercussões/aspectos envolvidos na obesidade e de suas implicações físicas, pois, embora os psicólogos possam atender a uma demanda psíquica, não se pode perder de vista a etiologia física, que muitas vezes é indevidamente interpretada. Também é da responsabilidade do psicólogo a criteriosa seleção das técnicas e recursos específicos próprios. Assim, parece 
que o profissional da psicologia, entre outros profissionais que trabalham com esta parcela da população, tem o desafio de desenvolver instrumentos específicos (muitos já disponíveis em outras culturas) para o paciente obeso. $\mathrm{O}$ estudo de intervenções mais eficazes para a promoção de saúde e bem-estar psicológico também está recomendado.

\section{REFERÊNCIAS}

Amorin, P. (2000). Mini International Neuropsychiatric Interview (MINI): validação de entrevista breve para diagnóstico de transtornos mentais. Revista Brasileira de Psiquiatria, 3(22), 106115.

Appolinário, J. C, Coutinho, W., \& Povoa, L. C. (1995). O transtorno do comer compulsivo: revisão da literatura. Jornal Brasileiro de Psiquiatria, 44(supl.1), S38-S45.

Antone, G. J., Lord, R. V. N., Demeester, T. R., \& Crookes, P. F. (2003). The duodenal switch operation for the treatment of morbity obesity. Annals of surgery, 238, 618-628.

Altfas, J. R. (2002). Prevalence of attention deficit/hyperactivity disorder among adults in obesity treatment. BMC Psychiatry, 2, 19.

Baskin M. L., Ard J, Franklin F., \& Allison D.B. (2005). Prevalence of obesity in the United States. Obesity Review, 6(1):5-7.

Becker, E. S., Margraf, J, Turke, V, Soeder, U \& Neumer, S. (2001). Obesity and mental illness in a representative sample of young women. International Journal of Obesity and Related Metabolic Disorder, 25(Suppl 1), S5-S9.

Braguinsky, J. (2002). Obesity prevalence in Latin America. Anales, 25(Suppl 1), 109-115.

Konok, G.(1999). The obesity adjustment survey: development of a scale to assess psychological adjustment to morbid obesity. International Journal of Obesity, 23, 505-511.

Carpenter, K. M., Hasin, D. S., Alisson, D. B., \& Faith M. S. (2000). Relationships between obesity and DSM-IV major depressive disorder, suicide ideation, and suicide attempts: results from a general population study. American Journal Public Health, 90, 251-257.

Ciconelli, R. M. (1997). Tradução para o português e validação do questionário genérico de avaliação de qualidade de vida "medical outcomes study 36-item short-form health suvey (SF-36)" Tese de Doutorado Não-Publicada, Escola Paulista de Medicina, Universidade Federal de São Paulo: São Paulo.

Claudino, A. M., \& Borges, M. B. F. (2002) Critérios diagnóstcos para os transtornos alimentares: conceitos em evolução. Revista de Psiquiatria Clínica, 24;(supl III), 7-12.

Coutinho, W. (1999). Consenso Latino-Americano Em Obesidade. Documento com redação abrangendo os mais diversos aspectos relacionados com o combate à obesidade, como métodos diagnósticos e terapêuticos, medidas preventivas de saúde pública e estratégias de vigilância ética na abordagem do paciente obeso. Arq Bras de End e Metab, 43(1),21-67.

Cordás, T., \& Neves, J. (1999). Escala de avaliação de transtornos alimentares. Revista de Psiquiatria Clínica ,26, 41-47.
Crippa, J. A. S., Hallar, J. E. C., Vilela, J. A. A., Loureiro, S. R., \& Zuardi, A. W. (1999). A escala breve de avaliação psiquiátrica (Brief Psychiatric Ratin Scale, BPRS): uma revisão. Jornal Brasileiro de Psiquiatria, 8, 355-361.

Cunha, J.A. (2000) Psicodiagnóstico V. Porto Alegre: Artmed.

Devlin, M. J., Yanovski, S. Z., \& Wilson, G. T. (2000). Obesity: what mental health professionals need to know. American Journal of Psychiatry, 156, 854-866.

Dobrow, I., Kamenetz, C., \& Devlin, M. (2002). Aspectos Psiquiátricos da obesidade. Revista Brasileira de Psiquiatria, 24(suppl. 3), 63-67.

Duchesne, M. (1995). Transtornos alimentares. Em Rangé, B. (Org.), Psicoterapia Comportamental e Cognitiva de Transtornos Psiquiátricos. Rio de Janeiro: Psy II.

DSM-IV-TR. (2002). Manual diagnóstico e estatístico de transtornos mentais. (4.ed. revisada). Porto Alegre: Artmed.

Fabricatore, A., \& Wadden, T. (2004). Psychological aspects of obesity. Clinics in Dermatology, 22(4), 332-337.

Fairburn, C. G., \& Cooper, Z. (1993).The eating disorder examination. Em: Fairburn, C. G., Wilson, G. T. Binge eating: nature, assessment, and treatment. New York: Guilford Press.

Fairburn, C.G., Cooper, Z., Doll, H.A., Norman, P., \& O'Connor, M. (2000). The natural course of bulimia nervosa and binge eating disorder in young women. Archives of General Psychiatry, 57, 659-665.

First, M. B., Spitzer, Robert L, Gibbon Miriam, \& Williams, Janet B.W. (2002) Structured Clinical Interview for DSM-IV-TR Axis I Disorders, Research Version, Patient Edition. (SCID-I/P). New York: Biometrics Research, New York State Psychiatric Institute.

Freitas, S., \& Appolinário, J. C. (2001).Tradução e adaptação para o português da Escala de Compulsão Alimentar Periódica. Revista Brasileira de Psiquiatria, 23, 215-220.

Freitas, S., Gorenstein, C., \& Appolinário, J. C. (2002). Instrumentos para a avaliação dos transtornos alimentares. Revista Brasileira de Psiquiatria, 24, 34-38.

Greenway, F. L., \& Smith, S. R. (2000). The future of obesity. Research Ingestive Behavior and Obesity Nutrition, 16, 976-982.

IBEG; MINISTÉRIO DA SAÚDE, MINISTÉRIO DO PLANEJAMENTO; ORÇAMENTO E GESTÃO. (2004). Pesquisa de orçamentos familiares 2002-2003: análise da disponibilidade domiciliar de alimentos e do estado nutricional no Brasil. Rio de Janeiro.

John, U., Meyer, C., Rumpf, H. J., \& Hapke, U. (2005). Relationships of psychiatric disorders with overweight and obesity in an adult general population sample. Obesity Research,13, 101-109.

Kendrick, T. (1996). Cardiovascular and respiratory risk factors and symptoms among general practice patients with long-term mental ilness. The British Journal of Psychiatry, 169, 733-739.

Lamertz, C. M., Jacobi, C., Yassouridis, A., Arnold, K., \& Henkel, A.W. (2002). Are obese adolescents and young adults at higher risk for mental disorder? A community survey. Obesity Research,10, 1152-1160.

Le Pen C., Levy E., Loos F., Banzet M. N., \& Basdevant A. (1998). "Specific" scale compared with "generic" scale: a double measurement of the quality of life in a French community sample of obese subjects. Journal of Epidemiology and Community Health, 52, 445-450. 
Maddi, S. R., Knoshaba, D. M., Persico, M., Bleecker, F., \& VanArsdall, G. (1997) Psychosocial correlates of psychopathology in a national sample of the morbidly obese. Obesity surgery, 7, 397- 404.

Marini, E. (2002). A Opção dos Supergordos. Isto é, São Paulo, n.1718.

Matos, M. I., \& Zanella, M. T. (2002). Alterações do comportamento alimentar, ansiedade, depressão e imagem corporal em pacientes com obesidade grau III. Abeso. Ano III. (9).

Mattos, A. F. G. (2004). Cirurgia bariátrica: mitos e verdades. Recuperado em 26 de outubro de 2004 em http:// www.sbem.org.br

Mattos, P., Saboya, E., Ayrão, V., Segenreich, D., Duchesne, M., \& Coutinho, G. (2004). Transtornos alimentares comórbidos em uma amostra clínica de adultos com transtorno do déficit de atenção com hiperatividade. Revista Brasileira de Psiquiatria, 26, 248-250.

Mitchell, J. E., \& Mussell, M. P. (1995). Comorbidity and binge eating disorder. Addict Behavior, 20, 725-732.

Parahoo, K. (2005). Review of methods for testing and evaluating survey questionnaires. Biomed Engineering Online, 4:3.

Pareja, J. C. (2002). Obesidade Mórbida. Recuperado em 06 de novembro de 2002, em http:// www.sppc.med.br

Pasquali, L. (org). (2001). Técnicas de exame psicológico -TEP: fundamentos das técnicas psicológicas. São Paulo: Casa do Psicólogo/ Conselho Federal de Psicologia.

Pope, H. G. Jr., et al (2006). Binge eating disorder: a stable syndrome. American Journal of Psychiatry, 163(12), 2181-2183.

Roberts, R. E., Deleger, S., Strawbridge, W. J., \& Kaplan, G. A. (2003). Prospective association between obesity and depression: evidence from Alameda County Study. International journal of Obesity. 27, 514-521.

Segal, A. (2004). Obesidade não tem cura mas tem tratamento. Rio de Janeiro: Ediouro.
Sheehan, D. V., et al. (1997). Reliability and Validity of the MINI International Neuropsychiatric Interview (M.I.N.I.): According to the SCID-P. European Psychiatry, 12, 232-241.

Strawbridge, W. J., Deleger, S., Roberts, R. E., \& Kaplan, G. A. (2002). Physical activity reduces the risk of subsequent depression for older adults. American Journal Epidemiology, 156, 328-334.

Stunkard, A. J., \& Wadden, T. A. (1992). Psychological aspects of severe obesity. American Journal of Clinical Nutrition, 55(2 suppl), 524S-532S.

Sturm, R., \& Wells, K. B. (2001). Does obesity contribute as much to morbity as poverty or smoking? Public Health,115, 229-235.

Tauber, G. (1998). Obesity: how big a problem? Science.

Wilfley, D. E., Schawartz, M. B., Spurrell, E. B., \& Fairburn, C. G. (2000). Comorbid psychopathology in binge eating disorder: relation to eating disorder severity at baseline and following treatment. Journal Consult Clinic Psychology, 68, 641- 649.

Womble, L. G., Williamson, D. A., Greenway, F. L., \& Redmann, S. M. (2002). Psychological and behavioral predictors of weight loss during drurg treatment for obesity. International Journal of Obesity, 25, 340-345.

Wonderlich, S., Joiner, Jr., T., Keel, P., Williamson, D., \& Crosby, R. (2007). Eating disorder diagnoses: empirical approaches to classification. American Psychologist. 62(3), 167-180.

Yanovsky, S. Z., Nelson, J. E., Dubbert, B. K., \& Spritzer, R. L. (1993). Association of Binge Eating Disorder and psychiatric comorbidity in obese subjects. American Journal of Psychiatry, 10, 1472-1479.

Yanovski, J. A., \& Yanovski, S. Z. (1999). Recent advances in basic obesity research. Contempo, 282, 1504-1506.

Recebido em 23/02/2007 Aceito em 21/12/2007

Endereço para correspondência :

Fabiana Silva Costa. Av. Protásio Alves, 1032/401, CEP 90410-004, Porto Alegre - RS, Brasil. E-mail: fabianascosta@gmail.com 